Meta

Journal des tradlucteurs

Translators' Journal

\title{
Quelques termes de soudage
}

\section{Bruno Couture}

Volume 17, numéro 3, septembre 1972

URI : https://id.erudit.org/iderudit/002427ar

DOI : https://doi.org/10.7202/002427ar

Aller au sommaire du numéro

Éditeur(s)

Les Presses de l'Université de Montréal

\section{ISSN}

0026-0452 (imprimé)

1492-1421 (numérique)

Découvrir la revue

Citer cet article

Couture, B. (1972). Quelques termes de soudage. Meta, 17(3), 171-176.

https://doi.org/10.7202/002427ar d'utilisation que vous pouvez consulter en ligne.

https://apropos.erudit.org/fr/usagers/politique-dutilisation/ 


\section{QUELQUES TERMES DE SOUDAGE}

Certains termes fondamentaux du vocabulaire anglais du soudage, tels que brazing et soldering, présentent des difficultés de traduction dont plusieurs auteurs de dictionnaires bilingues ou multilingues, comme ceux du Elsevier de la métallurgie ${ }^{1}$, du Kettridge technique ${ }^{2}$ et, dans une moindre mesure, du Harrap ${ }^{3}$, ne semblent guère avoir été conscients. Le résultat, c'est que, au mot soldering, par exemple, ces ouvrages proposent comme équivalents français soudage et soudure, d'où l'on peut déduire que pour leurs auteurs, soldering est un parfait synonyme de welding. D'autres ouvrages du même genre, le Vocabulaire anglais-français de Cusset ${ }^{4}$, notamment, ne donnent même que des locutions composées de soldering, où celui-ci est invariablement rendu par à souder, ce qui, en définitive, revient au même.

Quant au mot brazing, Cusset le traduit par brasure et Kettridge, par brasage, brasement et brasure, mais dans ce dernier cas, l'auteur du Supplément a cru bon d'ajouter aussi soudo-brasure. C'est pourtant le Harrap qui apporte l'élément nouveau de nature à fournir un léger indice, car aux trois équivalents déjà relevés dans le corps du Kettridge il ajoute soudure (au laiton) et soudure forte, ce dernier terme étant du reste la seule traduction citée par l'Elsevier. Mis à contribution à son tour, le Lexique technique de Malgorn ${ }^{5}$ enregistre deux autres ex-

1. W. E. Clason, Elsevier's Dictionary of Metallurgy, New York, Elsevier Publishing Company, 1967.

2. J. O. Kettridge, English-French Dictionary of Technical Terms and Phrases, London, Routledge and Kegan Paul Ltd., 1967.

3. J. E. Mansion, Harrap's Standard French and English Dictionary, London, George G. Harrap and Co. Ltd., 1970.

4. Francis Cusset, Vocabulaire technique anglais-français français-anglais, Paris, BergerLevrault, 1965.

5. Guy Malgorn, Lexique technique anglais-français, Paris, Gauthier-Villars, 1969. 
pressions susceptibles de faire avancer la recherche : brazing or hard soldering (brasure, brasage) et soft soldering (brasage tendre). Le premier est d'ailleurs confirmé par le Dictionnaire technique de Belle-Isle ${ }^{6}$, qui donne cependant, au lieu du second, light brazing (brasure douce).

On s'étonnera donc moins, ensuite, de cette note liminaire du traducteur, dans la version française du Brazing Manual de l'American Welding Society : «Les termes américains n'ont pas été traduits, et ceci volontairement. Ils auraient apporté une confusion encore plus grande que celle qui existe déjà en français. Disons, en simplifiant, que, pour les Américains, welding = procédé d'assemblage endothermique ; arc welding = soudage à l'arc ; soldering = soudobrasage ; brazing = brasage (fort). En ce qui concerne le brasage tendre, il semble que la confusion persiste ${ }^{7}$.

Que cette démission du spécialiste n'empêche pas le profane de chercher à éclairer sa lanterne. Tout d'abord, puisqu'il est bien évident qu'il faut reprendre la question à ses débuts, pourquoi welding devrait-il se rendre par procédé d'assemblage endothermique, qui est bien plus une définition qu'une appellation? Ce que le traducteur du Brazing Manual a sans doute voulu faire ressortir, c'est que l'emploi du mot welding n'est pas limité au domaine de la métallurgie, contrairement à ce que peuvent laisser supposer certaines sources, où cette restriction de sens s'explique peut-être par le contexte général des ouvrages dans lesquels il figure (cf. Solders and Soldering, de Manko ${ }^{8}$, et le Dictionary of Metallurgy, de Birchon ${ }^{9}$ ). En effet, le World Book Dictionary définit ainsi le verbe to weld : "To join or combine (pieces of metal, plastic, etc.) by heating either by a flame torch or an electric current and hammering or pressing together while soft, or by melting a similar metal or plastic into the joint ${ }^{10}$. »Or, en dépit de la définition donnée par l'ouvrage de Meslier, la Soudure autogène au chalumeau et à l'arc ${ }^{11}$, qui, probablement pour la même raison, paraît restreindre d'une manière semblable le sens du mot soudure, il y a lieu de signaler qu'on trouve aussi ailleurs des définitions très larges de ce terme, par exemple celle du Grand Larousse encyclopédique, qui se lit comme suit : «Mode d'assemblage permanent de deux pièces métalliques ou de certains produits synthétiques, exécuté par voie thermique ${ }^{12}$.

Soit dit en passant, beaucoup d'auteurs francophones établissent, du moins implicitement, une distinction opportune entre soudage et soudure, et il serait logique d'en user de même avec brasage et brasure. Pour eux, « on appelle soudage l'opération qui consiste à assembler les matériaux, et soudure le résultat

6. Gérald-J. Belle-Isle, Dictionnaire technique général anglais-français, Québec, Bélisle éd., 1965.

7. American Welding Society, Manuel du brasage, Paris, Dunod, 1966, p. v.

8. Howard H. Manko, Solders and Soldering, Toronto, McGraw-Hill Book Company Inc., 1964 , p. 109.

9. D. Birchon, Dictionary of Metallurgy, London, George Newnes Limited, 1965.

10. The World Book Dictionary, Chicago, Field Enterprises Educational Corporation, 1971.

11. R. Meslier, la Soudure autogène au chalumeau et à l'arc, $8^{\mathrm{e}}$ éd., Paris, Eyrolles, «L'enseignement technique et professionnel ", 1968, p. 8.

12. Grand Larousse encyclopédique, Paris, Larousse, 1969. 
de cette opération ${ }^{1.3} \gg$. D'autre part, le lecteur attentif aura tout de suite remarqué une contradiction flagrante entre le Supplément du Kettridge technique ${ }^{14}$ et l'avantpropos du Brazing Manual ${ }^{15}$, pour qui le soudobrasage ou la soudobrasure correspondraient dans un cas au brazing et dans l'autre au soldering (à moins que ces deux termes anglais ne soient aussi des synonymes purs et simples). Le Dictionnaire trilingue des matériaux et des constructions ${ }^{16}$ ne commet-il pas une méprise semblable en faisant de soudo-brasage un équivalent aussi bien de brazing ou hard soldering que de braze welding?

En ce point de sa recherche, le traducteur généraliste, qui a fait le tour des dictionnaires techniques bilingues dont il dispose, est bien obligé de se tourner vers les encyclopédies et dictionnaires généraux pour tâcher d'y voir clair. Là encore, il ne tardera pas à se rendre compte que leurs articles sont loin de briller toujours par leur clarté. Ainsi, à côté d'indications utiles comme celle-ci : «... la brasure est encore appelée soudure forte... ${ }^{17}$ », le Grand Larousse fournit des précisions parfois contradictoires, du genre des suivantes : "La soudure autogène est exécutée à l'aide d'un chalumeau, le plus souvent oxyacétylénique ${ }^{18} \gg$; " la soudure à la forge est aussi une soudure autogène 19 »; «la soudure au chalumeau [est] fréquemment appelée soudure autogène, ou encore soudure oxyacétylénique... ${ }^{20}$. Pour sa part, le grand Robert mentionne simplement que la soudure autogène s'exécute "sans autre matière que les deux parties à souder 》, ajoutant en corollaire que la soudure hétérogène s'obtient «par l'intermédiaire d'un composé métallique ${ }^{21} »$. Enfin, pour le Dictionnaire encyclopédique Quillet ${ }^{22}$, qui a eu la bonne idée de dresser un tableau des différents procédés de soudure, il n'y a de soudures hétérogènes que les soudo-brasures, ces dernières étant soit des soudures tendres, soit des soudures fortes.

Convaincu désormais que seul le recours aux ouvrages spécialisés permettra de dissiper toute confusion, le traducteur prend peu à peu conscience que les équivoques découlent, pour une bonne part, de la polyvalence de certains termes qui sont pris tantôt comme génériques, tantôt comme spécifiques, suivant l'auteur qui les emploie et la langue qui les adopte. C'est bien l'impression qui se dégage de ce passage de l'Encyclopédie pratique de mécanique et d'électricité : "Cette évolution se fait au profit des méthodes d'assemblage par soudage autogène au sens le plus large du mot. Nous précisons à ce sujet que soudage autogène n'est pas synonyme de soudage oxyacétylénique ; ainsi, le soudage à l'arc électrique, le soudage par points - pour n'en citer que deux - sont aussi des procédés de

13. Henri Desarces, Encyclopédie pratique de mécanique et d'électricité, t. Mécanique, Paris, Librairie Aristide Quillet, 1965, p. 219.

14. Op. cit.

15. Op. cit.

16. J. Edwin Holmström, Maurice Fickelson, Danilo Jejcic, Dictionnaire trilingue des matériaux et des constructions/Trilingual Dictionary for Materials and Structures, Oxford, Pergamon Press, 1971, p. 443.

17. Op. cit., vol. 2 , p. 341 .

18. Op. cit., vol. 1, p. 748

19. Op. cit., vol. 1 , p. 749 .

20. Op. cit., vol. 9 , p. 924

21. Dictionnaire alphabétique et analogique de la langue française, Paris, Société du Nouveau Littré, 1969.

22. Dictionnaire encyclopédique Quillet, Paris, Librairie Aristide Quillet, 1970, vol. 7, p. 6389. 
soudage autogène ${ }^{23}$.» Les Techniques de l'ingénieur sont encore plus explicites à cet égard : «La terminologie française a conservé au terme soudure autogène son sens étymologique le plus général : est autogène toute soudure qui a été exécutée selon un processus qui a provoqué une modification, ou la disparition, du profil initial des pièces à assembler (par fusion, pression ou friction). Ainsi, au moins théoriquement, le terme autogène ne s'applique-t-il pas exclusivement au soudage aux gaz (comme c'est le cas dans d'autres pays et dans d'autres langues), mais aussi bien au soudage à l'arc, par résistance ou à d'autres procédés ${ }^{24}$.

Ces autres langues auxquelles il est fait allusion ici comprennent-elles l'anglais? La réponse est affirmative, s'il faut en croire les définitions qui suivent : «Autogenous welding, fusion welding without the addition of filler metal ${ }^{25} »$; «Autogenous welding, the joining of pieces of metal to other pieces of the same metal by fusion usually in a gas flame, sometimes without flux, but usually with a filler rod of the same metal ${ }^{26} »$; «Fusion welding GB, Autogenous welding, any welding process (whether manual, semi-automatic or automatic welding) in which the weld is made between metals in a state of fusion without hammering or pressure. British Standard 49927. . La contradiction qu'on relève entre les deux premières définitions, à propos de la non-intervention ou de l'addition habituelle d'un métal d'apport, montre que les francophones ne détiennent pas le monopole de la confusion dans ce domaine.

Quoi qu'il en soit, cet usage étranger peut expliquer le fait que certains auteurs de langue française limitent le champ du soudage autogène aux procédés par fusion. Tel est le cas de l'ouvrage de L. Ducros qui, en revanche, a le singulier mérite de mettre en lumière l'opposition fondamentale qui existe entre le soudage autogène et le brasage : «Le soudage autogène, dans lequel les éléments à assembler participent par fusion à la constitution du joint, se distingue donc sans équivoque possible du brasage, qui fournit un joint uniquement constitué grâce à l'intervention du métal d'apport, sans fusion du métal de base ${ }^{28}$. » De fait, ce sens générique du mot brasage est confirmé indirectement par les Techniques de l'ingénieur : " Dans le cas d'une matière métallique, c'est-à-dire dotée de la structure cristalline, c'est à l'échelle de cette structure qu'il s'agit de réaliser la continuité, mais cela n'implique pas nécessairement l'homogénéité quant à la composition chimique (cas des soudures dites hétérogènes ou des brasures) ${ }^{29}$, $\gg \mathrm{Le}$ Manuel pratique de Mendel étant encore plus clair à ce sujet, il mérite d'être cité assez longuement : «Les procédés de soudage hétérogène peuvent être réunis sous le nom général de brasage [...] On tend aujourd'hui à ajouter à la définition générale du brasage une condition supplémentaire, c'est que la température de fusion

23. Op. cit., t. Mécanique, p. 220.

24. Métallurgie, Paris, Techniques de l'ingénieur, 1969, p. 880-882.

25. Birchon, op. cit.

26. John S. Scott, A Dictionary of Civil Engineering, $2^{\mathrm{e}}$ éd., Harmondsworth, Penguin Books, 1967.

27. Dictionnaire trilingue des matériaux et des constructions, p. 452.

28. L. Ducros, le Soudage manuel à l'arc électrique, Paris, Editions J.-B. Baillière et Fjls, "Bibliothèque professionnelle $», 1971$, p. 6.

29. Op. cit., p. $880-882$. 
complète de l'alliage d'apport soit supérieure à $425^{\circ} \mathrm{C}$. Cette limitation vers le bas a pour but de rejeter dans une autre catégorie de procédés ceux que les Anglo-Saxons appellent soldering et que nous appelons soudure tendre ou, plus logiquement, brasage tendre [...] Deux sous-groupes de procédés répondant à la définition précédente du brasage peuvent être distingués : le soudo-brasage, procédé dérivé du soudage autogène [...] et le brasage par capillarité, dit encore brasage fort, que l'on considère actuellement comme le brasage proprement dit, [où] l'intervention des phénomènes de capillarité est essentielle ${ }^{30}$. 》

Le Dictionnaire de métallurgie de Bader et Théret ${ }^{31}$ distingue lui aussi deux grandes catégories de procédés de soudage, qu'il désigne respectivement sous le nom de soudure indirecte d'une part, et soudure directe ou autogène d'autre part. Il n'est pas sans intérêt pour le lexicographe de noter au passage ces variantes, car elles sont susceptibles d'aider à la compréhension des termes communément admis.

Il semble toutefois que l'anglais ne dispose pas de termes dont l'aire sémantique couvre entièrement celle des génériques français soudage autogène et brasage, d'où l'embarras des lexicographes, surtout lorsqu'ils font des dictionnaires anglais-français ou français-anglais dépourvus de définitions. C'est ainsi que, dans ses recueils terminologiques multilingues ${ }^{32}$, l'Institut international de la soudure, qui avait pourtant senti le besoin de justifier par une note définitoire l'emploi de soudobrasage en anglais d'Angleterre, donne à welding les sens de soudage autogène et de soudage, ce qui, pour le premier terme, appellerait au moins une explication. Pour sa part, l'excellent Vocabulaire de la Commission électrotechnique internationale ${ }^{33}$ traduit soudage par welding, brazing and soldering, mais il ajoute, à la fin de sa définition anglaise, cette observation complémentaire : "When the filler material is copper or copper-silver alloy the operation is usually referred to as brazing. When the filler material is tin or a lead-tin alloy it is usually referred to as soldering. »

Tout comme le Dictionnaire encyclopédique Quillet ${ }^{34}$, le traité de Meslier ${ }^{35}$ divise les procédés de soudage autogène en deux catégories : le soudage par fusion et le soudage par pression. Ici encore, il convient de noter que les spécialistes ne semblent pas s'entendre sur ce qu'on doit faire entrer dans chacune. En effet, selon les Techniques de l'ingénieur, «le soudage par pression englobe les procédés où la liaison est obtenue sans fusion, par une pression qui s'exerce à froid ou à chaud, ou avec fusion et pression ${ }^{36}$ », tandis que d'après le Vocabulaire électrotechnique international, le soudage par pression est un * soudage comportant une pression pour assurer l'exécution du joint, dont aucune partie

30. L. Mendel, Manuel pratique du soudage au chalumeau et d'oxycoupage, Paris, Dunod, 1970 , p. 199 et 200

31. Olivier Bader et Michel Théret, Dictionnaire de métallurgie, Paris, Eyrolles, 1962, p. 609.

32. Institut international de la soudure, Recueil terminologique multilingue du soudage et des techniques connexes : Termes généraux, Bâle, Société suisse de l'acétylène, 1955.

33. Commission électrotechnique internationale, Vocabulaire électrotechnique international, Groupe 40 : Applications électrothermiques, Genève, Bureau central de la C.E.I., 1960.

34. Op. cit.

35. Meslier, la Soudure autogène au chalumeau et à l'arc, p. 8 et 9.

36. Op cit., p. 880-883. 
constitutive n'a subi de fusion ${ }^{37} \gg$. Dans ce cas, ce qui complique un peu les choses, c'est donc l'existence de procédés de soudage mixtes, dont le classement varie selon le point de vue où l'on se place. Aussi n'est-il pas tenu compte de cet aspect du problème, dans l'essai de classification bilingue ci-dessous, établi d'après les données dont il a été fait état aux paragraphes précédents. Les désignations considérées comme des variantes y figurent entre parenthèses.

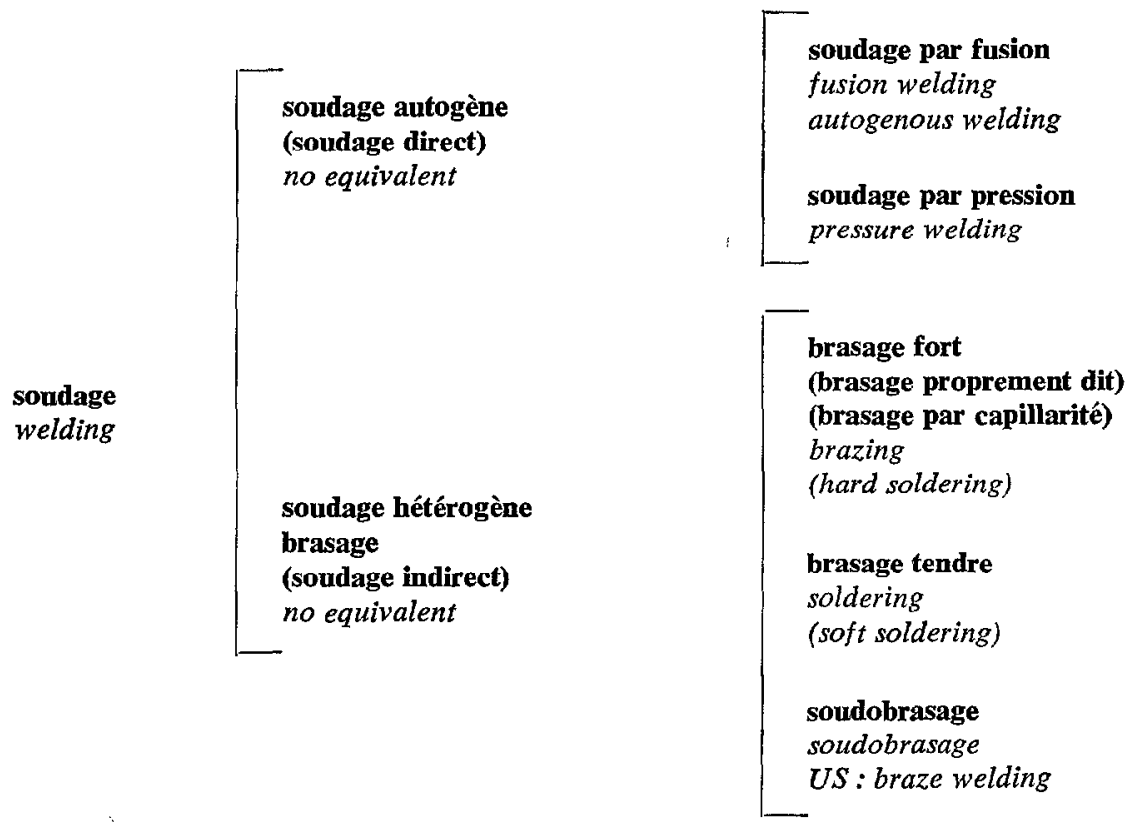

Nos exemples, empruntés au domaine du soudage, illustrent le fait qu'encore aujourd'hui, ainsi que l'écrivait $M$. Jean Darbelnet dans la revue Langages, les dictionnaires bilingues «ne mettent pas l'usager suffisamment en garde contre les méprises qui résultent de la non-superposition des aires sémantiques entre des mots qui en fait ne se correspondent qu'en partie ${ }^{38} \gg$. Il faudrait donc, comme le préconise ce distingué linguiste, que les lexicographes, mettant à profit les récents apports de la lexicologie différentielle, fassent un emploi plus systématique des marques d'usage sémantiques, en particulier celle du générique et du spécifique, qui est un cas particulier de l'extension. C'est à cette condition que les dictionnaires bilingues joueront vraiment leur rôle, évitant ainsi à l'usager les recherches nécessaires pour cerner le sens exact des termes dont traitent leurs articles.

Bruno Couture

37. Op. cit.

38. Jean Darbelnet, "Dictionnaires bilingues et lexicologie différentielle », Langages, Paris, Didier-Larousse, $\mathrm{n}^{\circ} 19$ (septembre 1970), p. 92. 